\title{
Effect of high flux plasma exposure on the micro-structural and -mechanical properties of ITER specification tungsten
}

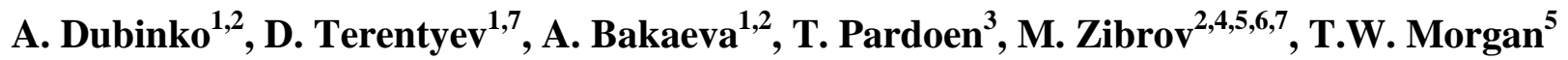 \\ ${ }^{1}$ Institute for Nuclear Material Sciences, SCK·CEN, $2400 \mathrm{Mol}$, Belgium \\ ${ }^{2}$ Department of Applied Physics, Ghent University, 9000 Ghent, Belgium \\ ${ }^{3}$ Institute of Mechanics, Materials and Civil Engineering, Université catholique de Louvain, Place \\ Sainte Barbe 2 L5.02.02, 1348 Louvain-la-Neuve, Belgium \\ ${ }^{4}$ Max-Planck-Institut für Plasmaphysik, Boltzmannstraße 2, D-85748 Garching, Germany \\ ${ }^{5}$ FOM Institute DIFFER - Dutch Institute for Fundamental Energy Research, De Zaale 205612 \\ AJ Eindhoven, the Netherlands \\ ${ }^{6}$ Physik-Department E28, Technische Universität München, James-Franck-Straße 1, D-85748 \\ Garching, Germany \\ ${ }^{7}$ National Research Nuclear University MEPhI (Moscow Engineering Physics Institute), \\ Kashirskoe shosse 31, 115409 Moscow, Russia \\ E-mail: adubinko@sckcen.be
}

\begin{abstract}
We have performed a combined study using transmission electron microscopy (TEM), nuclear reaction analysis (NRA) and nano-indentation (NI) techniques to reveal the impact of high flux plasma exposure on the properties of a sub-surface region of the commercially available pure tungsten fabricated following the ITER specification. TEM examination revealed the formation of a dense dislocation network and dislocation tangles, resulting in a strong increase in the dislocation density by at least one order of magnitude as compared to the bulk density. The plasma-induced dislocation microstructure vanishes within a depth of about 10-15 $\mu \mathrm{m}$ from the top of the exposed surface. Surface hardness after the plasma exposure was characterized by NI and was found to increase significantly in the sub-surface region of $1.5-3 \mu \mathrm{m}$. That was attributed to the resistance of the plasma-induced dislocation networks and deuterium-induced defects, whose presence within a depth of $\sim 1 \mu \mathrm{m}$ was unambiguously detected by the NRA measurements as well.
\end{abstract}

Keywords: high flux plasma, tungsten, dislocations. 


\section{Introduction}

Tungsten (W) is selected to be the material to produce plasma-facing components for ITER divertor and is also a candidate for the first wall material for DEMO [1]. The confinement of plasma in the ITER chamber is a technologically complicated process and in certain operational modes oscillations in plasma stability will inevitably cause so-called Edge Localized Modes (ELMs) [2]. Consequently, the plasma-facing material will experience thermal fatigue loading, which imposes a risk on the structural integrity because of crack nucleation and propagation. Hence, thermal, mechanical and microstuctural properties of tungsten are a central focus of investigations involving the plasma-material interaction (see recent review [3]). One particular problem that is currently being addressed is the combined nature of the high flux plasma consisting of both particle and thermal exposure. While transient heating invokes thermal stresses, the impact of hydrogen isotopes causes the formation of blister-like structures and/or bubbles further reducing the ductility and thermal conductivity of material in the sub-surface region. The evolution of the mechanical properties in the sub-surface region is therefore important for understanding the conditions favoring the nucleation of cracks [4].

Data available in literature show that under low-energy high flux deuterium plasma exposure (DPE) the concentration of retained deuterium (D) sharply decreases within the first several $\mu \mathrm{m}$ (see e.g. [5-9]). Increasing the exposure temperature and fluence results in the diffusion of plasma components deeper in the bulk so that the depth deposition consists of three zones: (i) the top-surface implantation layer (up to a depth of tens to hundreds of nano-meters), (ii) the sub-surface layer (up to several $\mu \mathrm{m}$ ), and (iii) the bulk (from about $10 \mu \mathrm{m}$ up to millimeters), as was recently discussed in [10]. However, it is expected that the high flux DPE at temperatures of $600 \mathrm{~K}$ and below primarily results in top- and sub-surface retention, because the diffusion of deuterium is limited by the material's microstructure (dislocations and grain boundary) [11, 12].

TEM investigation of the surface microstructure of a heavily deformed tungsten was recently performed in [13]. The pre-deformed tungsten was exposed to high flux plasma at $620 \mathrm{~K}$. In the exposed sample, a strong increase of the dislocation density in the sub-surface layer (i.e. within $\sim 10 \mu \mathrm{m}$ ) was found. In addition to the formation of dislocation pile-ups and networks, the presence of dislocation loops with Burgers vector $\mathrm{a}_{0} / 2\langle 111\rangle$ and $\mathrm{a}_{0}\langle 100\rangle$, typically observed under heavy ion or neutron irradiation [14], were identified. Unfortunately, complementary characterization of micro-mechanical properties was not performed prior outgassing and the impact of these microstructures on the hardness remains unknown.

To remove this gap, here, we perform a combined microstructural and micro-mechanical characterization using transmission electron microscopy (TEM), nuclear reaction analysis (NRA) and nano-indentation (NI) techniques to reveal the impact of the high flux low energy DPE on the properties of the sub-surface region of tungsten. The investigated tungsten grade (henceforth 
referred to as "IGP W", coming from ITER Grade Plansee Tungsten) was produced according to ITER specification in large quantities and distributed among different EU labs for a variety of tests including mechanical characterization, high heat flux properties, retention and response to ion and neutron irradiation in the framework of EUROfusion activies.

\section{Experimental procedure and reference microstructure}

The investigated samples, of size $10 \times 10 \times 1 \mathrm{~mm}$, were cut from a bar with a square crosssection of $36 \mathrm{~mm}$ made of tungsten of $99.99 \mathrm{wt} . \%$ purity. The bar was fabricated by hammering on both sides. The grains are therefore elongated along the bar axis and the square samples with dimensions $10 \times 10 \times 1 \mathrm{~mm}$ were cut such that the plasma beam would hit in the transverse direction with respect to the elongation of grains. The cut samples were annealed at $1273 \mathrm{~K}$ for one hour in an inert environment to remove the damage induced by the electric discharge machining (EDM). Transmission electron microscopy and scanning electron microscopy (SEM) following this shows the annealing did not alter the original texture of the material. Electron back scattering diffraction (EBSD) revealed that grains are randomly orientated and elongated with a size of 5-20 $\mu \mathrm{m}$ and 10-100 $\mu \mathrm{m}$, normal and along to the bar axis, respectively. TEM measurements show sub-grains are also elongated and their size varies in the range $0.6-1.7 \mu \mathrm{m}$ and $2.3-4 \mu \mathrm{m}$ in the normal and along the elongation directions, respectively. The samples for plasma exposure and subsequent NI testing were mechanically polished down to $0.25 \mu \mathrm{m}$ roughness to reach a mirror surface finish.

The high flux DPE was performed at the linear plasma generator Pilot-PSI $[15,16]$, which delivers high-density D plasmas mimicking the 'sub-displacement threshold' plasma wall interaction conditions expected in ITER. The energy of the D ions was $\sim 50 \mathrm{eV}$, achieved by the bias voltage, while imposing more than $900 \mathrm{eV}$ is required to initiate atomic displacement in $\mathrm{W}$. Although the plasma beam was non-uniform (full width at half maximum, FWHM, $10 \mathrm{~mm}$ ), the relatively small sample surface (approximately $9 \times 9 \mathrm{~mm}$ ) ensured a limited temperature gradient across the surface during the exposure, as measured and confirmed by an infra-red camera (FLIR A645 sc). The target exposure temperature was $473 \mathrm{~K}$ and the temperature variation was about $\pm 30 \mathrm{~K}$ across the surface, similar to what has been reported in [17]. The maximum particle flux in the centre of the sample was $\sim 10^{24} \mathrm{D} / \mathrm{m}^{2} / \mathrm{s}$. The flux was estimated according to the Bohm criterion from the plasma electron density and electron temperature, which were measured by Thomson scattering at a distance of about $2 \mathrm{~cm}$ from the target [18]. The samples were exposed to a fluence of $F \sim 5 \times 10^{25} \mathrm{D} / \mathrm{m}^{2}$.

After the plasma exposure and before TDS, the NRA measurements were performed in the central part of the sample. To determine the amount and depth distribution of retained D in the sub-surface region, nuclear reaction analysis (NRA) was used. The $\mathrm{D}\left({ }^{3} \mathrm{He}, \mathrm{p}\right) \alpha$ nuclear reaction was used by exposing the sample to a ${ }^{3} \mathrm{He}$ beam at multiple energies $(0.5,0.69,1.2,1.8$, $2.5,3.2,4.5 \mathrm{MeV})$ to achieve information from different depth regions. The shape and intensity 
of the proton spectrum for each ${ }^{3} \mathrm{He}$ beam energy was analysed and fitted. The reconstruction of depth profiles was performed with the use of the software packages SimNRA [19] and NRADC [20].

\section{Results}

The initial microstructure in terms of dislocation density and grain size was characterized by TEM and EBSD techniques. As mentioned in Section 2, the measurements performed in the as-received material (after removal of EDM damage by annealing at $1273 \mathrm{~K}$ ) revealed the average dislocation density to be $6 \times 10^{12} \mathrm{~m}^{-2}$. TEM observations showed that dislocation segments were nearly ubiquitously present within sub-grain interiors. No dislocation networks or pile-ups were observed, proving that the material is stress-relieved. These TEM-observable microstructural features are shown in Fig.1.

Importantly, to provide adequate comparison between the bulk and surface microstructure, the TEM examination was performed using back-side electrochemical polishing for both reference and as-exposed samples. The top surface of the as-received sample was mirrorfinished before the exposure and no further polishing on the exposed surface was applied to avoid any post-exposure damage. The reference sample was characterized by exactly the same procedure. TEM lamella were cut so as to observe the microstructure normal to the plasma exposure.

TEM observations, made in the immediate (implantation) region (as the depth resolved by TEM is within 100-150 nm), showed a significant increase in dislocation density, as shown in Fig.2. The computed dislocation density was found to be by one order of magnitude higher in the first sub-surface region $\left(6 \times 10^{13} \mathrm{~m}^{-2}\right)$ of the exposed sample as compared to that in the bulk $\left(6.5 \times 10^{12} \mathrm{~m}^{-2}\right)$. Statistical analysis was performed by measuring the dislocation density in $15-20$ different sub-grains and the standard error does not exceed $25 \%$ of the absolute value.

In addition to the dislocation lines and tangles, a pattern of elongated sub-grains is clearly seen, which means that the grain structure was not affected by the plasma exposure. The interface of sub-grains, seen to result in a sharp contrast in the reference sample, has become broadened after the plasma exposure, comparing Fig.1(c) and Fig.2(c). We interpret this as the impact of thermal stress which disturbed the original arrangement of the dislocation walls forming the interface of the low angle grain boundaries.

To investigate the microstructure in the deeper sub-surface region, back-side electrochemical polishing was applied to retain the exposed surface and was followed by consecutive electrochemical polishing from both sides for 4, 5, 10,15 and 23 seconds to remove approximately $3,7,10$ and $15 \mu \mathrm{m}$ of the exposed surface layer, without inducing mechanical damage on the plasma-exposed side.

The typical appearance of dislocations at the studied depths is shown by a set of pictures in Fig.3, on its right-hand side. The calculated dislocation density and the error bar are presented 
on the left-hand side of the figure. The origin of the variation of dislocation density is related to the variation in grain size as well as to the orientation of the grain. Evidently, the dislocation density decreases as the surface region is removed, reaching the value found in the unexposed sample at $\sim 15 \mu \mathrm{m}$ below the exposed surface.

While inspecting the dislocation microstructure, dislocation loops with "coffee bean" and "dot-like" contrasts were also regularly observed within $10 \mu \mathrm{m}$ depth, however, these defects will be characterized separately in future work.

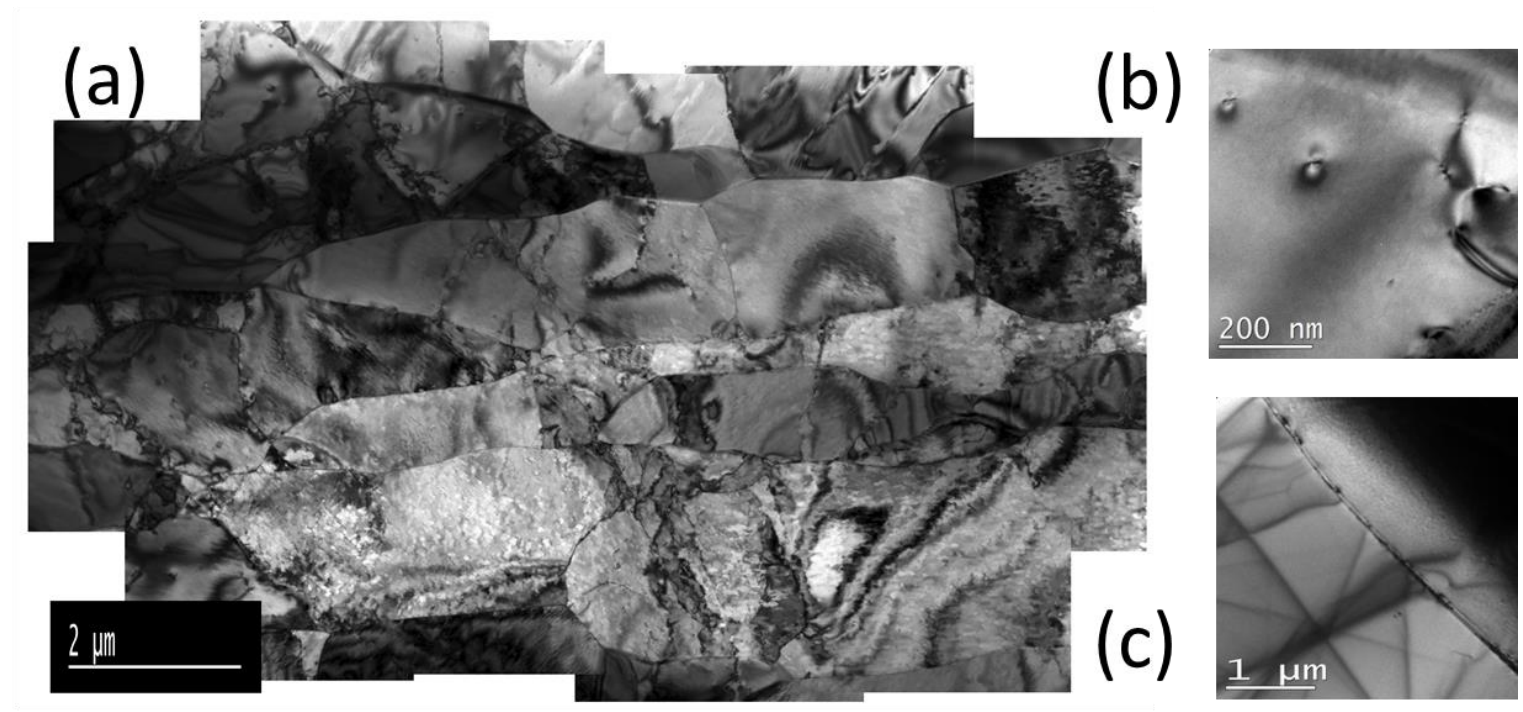

Fig.1. TEM micrograph of the typical bulk microstructure of the IGP W in the stress-relieved condition; (a) composite TEM image showing sub-grain microstructure: the grains and subgrains are elongated in the left-right direction; (b) example of dislocation lines identified within a sub-grain; (c) example of a low-angle grain boundary with a fine dislocation structure. 


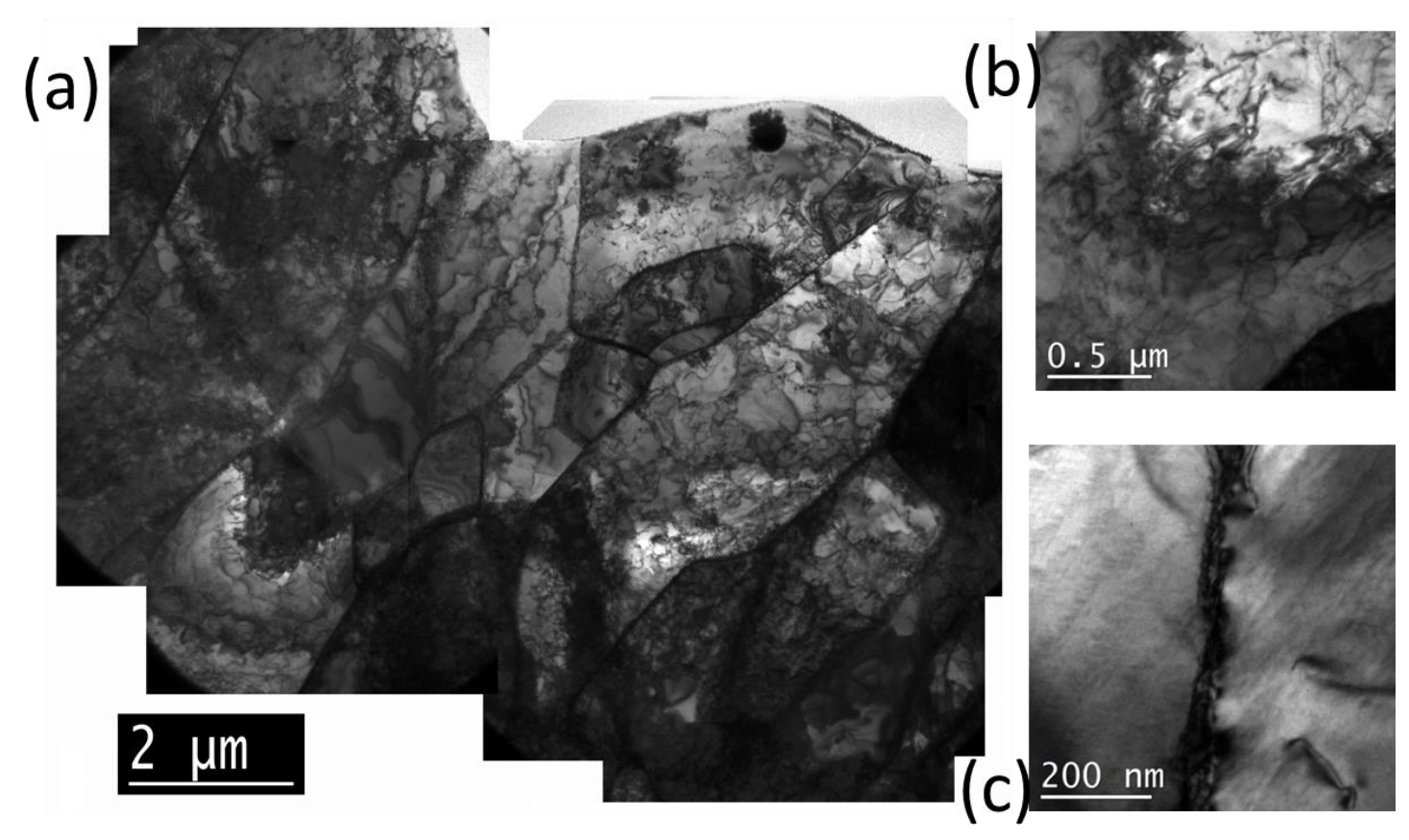

Fig.2. TEM micrograph of the surface microstructure of the exposed sample; The image shows a microstructure normal to the plasma beam. (a) composite TEM image showing sub-grain microstructure; (b) example of dislocation tangles identified within a sub-grain; (c) example of a low-angle grain boundary which is broadened as a result of the exposure.
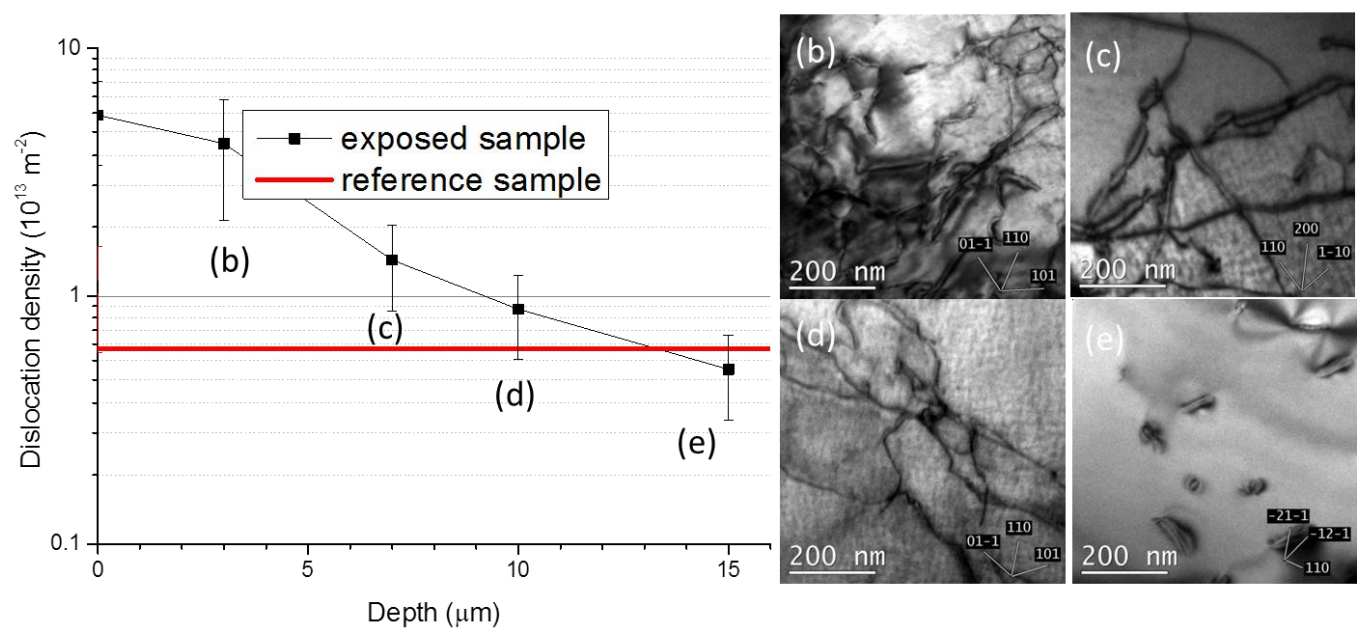

Fig.3. The left figure presents the average dislocation density as a function of depth from the exposed surface. The red line corresponds to the mean dislocation density measured in the reference stress-relieved sample. The right-hand side set of pictures show examples of TEM images used to measure the dislocation density following the removal of the exposed surface: (b) $3 \mu \mathrm{m}$ removed, (c) $7 \mu \mathrm{m}$ removed, (d) $10 \mu \mathrm{m}$ removed and (d) $15 \mu \mathrm{m}$ removed. 

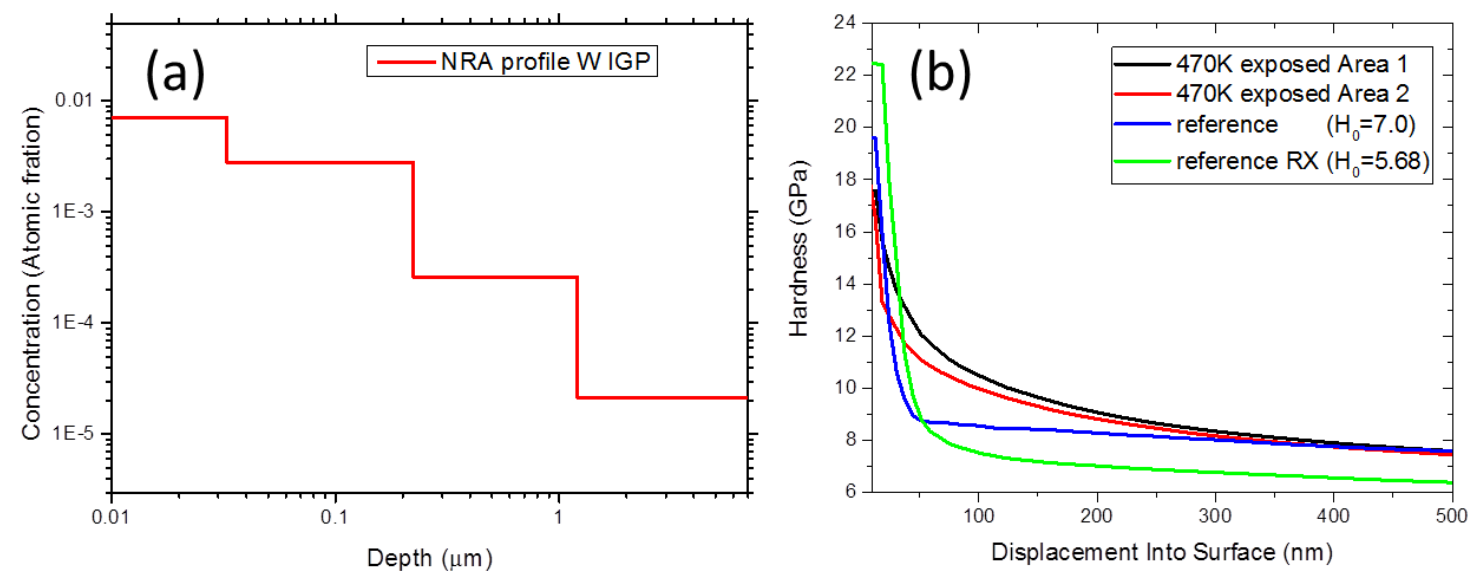

Fig.4. (a) NRA depth profile of retained D, measured in the centre sample and (b) hardness depth profile measured by nano-indentation; following the exposure at $473 \mathrm{~K}$ in Pilot-PSI up to a fluence of $\sim 5 \times 10^{25} \mathrm{D} / \mathrm{m}^{2} . \mathrm{H}_{0}$ is the saturated hardness, measured at the penetration depth of 1.5 $\mu \mathrm{m}$. "Reference" indicates the stress-relieved sample, while "Reference RX" is the stress-relieved sample additionally annealed at $1873 \mathrm{~K}$ for 1 hour to achieve recrystallized state.

The deuterium depth distribution, measured by NRA, shows a high deuterium concentration in a very narrow region close to the sample surface, see Fig.4(a). The majority of the NRA-detected D is located within $1 \mu \mathrm{m}$ from the top surface, while the maximum measured depth is $7 \mu \mathrm{m}$. Deeper into the bulk of the sample, the deuterium content decays exponentially and becomes comparable to the resolution of the techniques, when specifically applied to tungsten with the $4.5 \mathrm{MeV} \mathrm{He}^{3}$ beam [21].

To characterize the impact of the high flux DPE on the modification of mechanical properties, we also performed a set of nano-indentation measurements. The exposed and reference samples were tested using an Agilent G200 nano-indenter in order to measure the Young's modulus and hardness. The indentation measurements were performed in continuous stiffness mode (CSM) [22] with the standard XP head equipped with a Berkovich diamond tip. The oscillation amplitude and frequency were respectively $2 \mathrm{~nm}$ and $45 \mathrm{~Hz}$. The indentation strain rate was set to $0.05 \mathrm{~s}^{-1}$ and the specimens were indented down to a penetration depth $h_{N I}=1.5 \mu \mathrm{m}$. Two zones on the exposed sample were investigated by performing 25 indents spaced by $60 \mu \mathrm{m}$ between each zone. The hardness was calculated using the classical Oliver $\&$ Pharr method [22]. Calibration for the tip area function was performed by carrying out a series of indents in a reference fused silica sample. Nano-indentation was performed on three samples, namely: non-exposed IGP W, plasma-exposed IGP W and non-exposed but recrystallized IGP W (annealed at $1873 \mathrm{~K}$ in high vacuum for 1 hour). The measurements were performed in different areas preselected using an optical microscope to avoid apparent surface defects. 
The evolution of the hardness as a function of the indentation depth, obtained by averaging the results, is presented in Fig.4(b). For the recrystallized IGP W, the saturated hardness at a depth of $1.5 \mu \mathrm{m}$ reaches $5.68 \pm 0.12 \mathrm{GPa}$, which is in good agreement with $6 \mathrm{GPa}$ and 5.6 GPa reported for recrystallized polycrystalline $\mathrm{W}$ (of similar purity as studied here) by Armstrong et.al. [23] and by Zhang et.al. [24]. For the non-recrystallized IGP W, we clearly see an increase of the bulk hardness up to $7 \pm 0.1 \mathrm{GPa}$. This increase must be attributed to the presence of dislocations and low-angle grain boundaries, which offer extra resistance to the penetration of the indenter.

Nano-indentation measurements made in Ref. [23] and [24] show that mixed H-He implantation (by ion accelerator) and damage by $\mathrm{Fe}^{+}$ions leads to a pronounced increase of the hardness within the implantation range, which was about $\sim 1 \mu \mathrm{m}$. The hardness curves measured in the plasma-exposed sample are shown in Fig.4(b). Note that there is only a small deviation in the results obtained in the two probed areas indicating that the hardness profiles are likely quite uniform across the surface. In the present study, the kinetic energy of D ions was $\sim 50 \mathrm{eV}$, implying that the ion penetration depth is limited to several nanometers. However, the resulting increase of the hardness spreads over a penetration depth of about $300 \mathrm{~nm}$, meaning that the actual plasma-induced damage spreads to a distance of a factor of 5-10 larger, thus becoming 1.5$3 \mu \mathrm{m}^{1}$. This result clearly demonstrates that there is a spatial correlation between the increase of the hardness and depth distribution of D as measured by NRA techniques.

\section{Conclusive remarks}

To summarize, we performed a combined experimental study using TEM, NRA and nano-indentation techniques to investigate the microstructure and hardness of ITER-specification tungsten after the exposure to high flux plasma $\left(10^{24} \mathrm{D} / \mathrm{m}^{2} / \mathrm{s}\right)$ at $470 \mathrm{~K}$. The apparent increase in the dislocation density in the sub-surface region, revealed by TEM, should be attributed to the thermal stresses and subsequent plastic deformation, although the surface temperature was most likely below the ductile to brittle transition temperature (expected to be around 523-573 K for this particular grade). The reduction of the dislocation density to the bulk level beyond a depth of 15 $\mu \mathrm{m}$ correlates well with the strong gradient of the D concentration, as measured by NRA, and enhanced hardness, as measured by the nano-indentation. However, the extension of the D penetration and hardness increase is observed only within a depth of 1.5-3 $\mu \mathrm{m}$, while the exposure impact on the dislocation pattern is within $15 \mu \mathrm{m}$. We therefore suggest that, in the present experiment, the thermal stress primarily imposes the microstructural changes, which subsequently affect the process of deuterium diffusion and trapping. Nano-indentation testing clearly reveals the increase of the hardness, however, it does not allow to distinguish the

\footnotetext{
${ }^{1}$ The actual probed volume is larger than the indentation depth because the geometrically necessary dislocations generate under indenter tip and propagate in material.
} 
contributions from deuterium defects and stress-induced dislocation microstructure. Clearly, mode detailed analysis of the thermal stresses induced during the whole exposure cycle with the usage of finite element techniques is required.

\section{Acknowledgements:}

This project was carried out with financial support from NWO (Netherlands) and FOD (Belgium). This work was supported by the European Commission and carried out within the framework of the Erasmus Mundus International Doctoral College in Fusion Science and Engineering (Fusion-DC). The work was also partially supported by the funding received from the Euratom research and training programme 2014-2018 under grant agreement No 633053 (EUROfusion/Enabling Research programme). The views and opinions expressed herein do not necessarily reflect those of the European Commission.

\section{References:}

[1] M. Rieth, S.L. Dudarev, S.M.G. de Vicente, J. Aktaa, Et.al., Journal of Nuclear Materials, 432 (2013) 482-500.

[2] A. Loarte, G. Saibene, R. Sartori, M. Becoulet, L. Horton, T. Eich, A. Herrmann, M. Laux, G. Matthews, S. Jachmich, N. Asakura, A. Chankin, A. Leonard, G. Porter, G. Federici, M. Shimada, M. Sugihara, G. Janeschitz, Journal of Nuclear Materials, 313 (2003) 962-966.

[3] G. Pintsuk, Comprehensive Nuclear Materials, 4 (2012) 551-581.

[4] M.Y. Li, J.H. You, Fusion Engineering and Design, 101 (2015) 1-8.

[5] L. Buzi, G. De Temmerman, B. Unterberg, M. Reinhart, T. Dittmar, D. Matveev, C. Linsmeier, U.

Breuer, A. Kreter, G. Van Oost, Journal of Nuclear Materials, 463 (2015) 320-324.

[6] Y. Zayachuk, M.H.J. 't Hoen, P.A.Z. van Emmichoven, D. Terentyev, I. Uytdenhouwen, G. van Oost, Nuclear Fusion, 53 (2013) 013013.

[7] Y. Zayachuk, A. Manhard, M.H.J. t'Hoen, W. Jacob, P.A.Z. van Emmichoven, G. van Oost, Nuclear Fusion, 54 (2014) 123013.

[8] K. Schmid, V. Rieger, A. Manhard, Journal of Nuclear Materials, 426 (2012) 247-253.

[9] V.K. Alimov, B. Tyburska-Puschel, S. Lindig, Y. Hatano, M. Balden, J. Roth, K. Isobe, M. Matsuyama, T. Yamanishi, Journal of Nuclear Materials, 420 (2012) 519-524.

[10] T. Tanabe, Physica Scripta, T159 (2014).

[11] D. Terentyev, V. Dubinko, A. Bakaev, Y. Zayachuk, W. Van Renterghem, P. Grigorev, Nuclear Fusion, 54 (2014) 042004.

[12] D. Terentyev, G. De Temmerman, T.W. Morgan, Y. Zayachuk, K. Lambrinou, B. Minov, A. Dubinko, K. Bystrov, G. Van Oost, J Appl Phys, 117 (2015) 083302.

[13] A. Dubinko, A. Bakaeva, M. Hernandez-Mayoral, D. Terentyev, G. De Temmerman, J.M. Noterdaeme, Phys. Scripta, T167 (2015) 014030.

[14] A. Hasegawa, M. Fukuda, S. Nogami, K. Yabuuchi, Fusion Engineering and Design, 89 (2014) 15681572.

[15] G.J. van Rooij, V.P. Veremiyenko, W.J. Goedheer, B. de Groot, A.W. Kleyn, P.H.M. Smeets, T.W. Versloot, D.G. Whyte, R. Engeln, D.C. Schram, N.J.L. Cardozo, Applied Physics Letters, 90 (2007).

[16] G. De Temmerman, e. al., Nuclear Fusion, 51 (2011) 073008.

[17] M.H.J. 't Hoen, B. Tyburska-Pûschel, K. Ertl, M. Mayer, J. Rapp, A.W. Kleyn, P.A. Zeijlmans van Emmichoven, Nuclear Fusion, 52 (2012) 023008. 
[18] H. van der Meiden, R. Al, C. Barth, A. Donee, R. Engeln, W. Goedheer, Rev. Sci. Instrum. , 79 (2008) 013505.

[19] M. Mayer, SimNRA User Guide, Report IPP 9/113, Max-Planck-Institut für Plasmaphysik, Garching, Germany, (1997).

[20] K. Schmid, U. von Toussaint, Nuclear Instruments \& Methods in Physics Research B, 281 (2012) 6471.

[21] M. Mayer, E. Gauthier, K. Sugiyama, U. von Toussaint, Nucl Instrum Meth B, 267 (2009) 506-512.

[22] W. Oliver, G. Pharr, J Mater Res, 19 (2004) 3-20.

[23] D.E.J. Armstrong, P.D. Edmondson, S.G. Roberts, Applied Physics Letters, 102 (2013).

[24] Z.X. Zhang, D.S. Chen, W.T. Han, A. Kimura, Fusion Engineering and Design, 98-99 (2015) 2103-2107. 